\title{
Biomechanical and in vivo evaluation of experimental closure devices of the annulus fibrosus designed for a goat nucleus replacement model
}

\author{
Johannes L. Bron • Albert J. van der Veen • Marco N. Helder • \\ Barend J. van Royen · Theodoor H. Smit • Skeletal Tissue Engineering Group Amsterdam • \\ Research Institute MOVE
}

Received: 20 September 2009/Revised: 16 January 2010/Accepted: 9 March 2010/Published online: 17 April 2010

(C) The Author(s) 2010. This article is published with open access at Springerlink.com

\begin{abstract}
Promising strategies are being developed to replace or regenerate the herniated nucleus pulposus. However, clinical efficacy of these methods has still to be addressed, and the lack of appropriate annulus closure techniques is increasingly being recognised as a major limiting factor. In the current study, in vitro and in vivo evaluation of novel annulus closure devices (ACDs) was performed. These devices are intended to be used in adjunct to nucleus replacement therapies in an experimental goat study. After a standardised discectomy had been performed, different ACDs were implanted solely or in addition to a collagen nucleus replacement implant. Biomechanical effects and axial failure load were assessed in vitro and followed by in vivo evaluation in a goat model. On axial compression, the average axial failure load for ACDs with four barb rings was significantly higher compared to the implants with five barb rings. The increased range of flexion-extension and latero-flexion observed after discectomy were restored to the normal range after implantation of the implants. Positive findings with the four-ring ACD were confirmed in goats after a follow-up of 2 weeks in vivo. However, after 6 weeks most implants $(n=16)$ showed signs of destruction and displacement. Although there seemed to be a tendency towards better results when ACDs were placed in addition to the nucleus replacements, these differences were not statistically significant. Moreover, two endplate reactions extending into
\end{abstract}

J. L. Bron · M. N. Helder · B. J. van Royen · T. H. Smit ( $\varangle)$ Department of Orthopaedic Surgery, VU University Medical Center, Postbox 7057, 1007 MB Amsterdam, The Netherlands e-mail: th.smit@vumc.nl

A. J. van der Veen

Department of Physics and Medical Technology,

VU University Medical Center, Amsterdam, The Netherlands the subchondral bone were observed, most likely due to continuous friction between the ACD and the vertebrae. Although current results are encouraging first steps towards the development of an efficient ACD for animal models, further optimisation is necessary. Current results also show that one cannot rely on in vitro biomechanical studies with annulus closure techniques, and these should always be confirmed in vivo in a large animal model.

Keywords Annulus closure device .

Nucleus replacement $\cdot$ Spine $\cdot$ Goat model

\section{Introduction}

The lack of sufficient strategies to deal with the damaged annulus fibrosus (AF) may currently be recognised as one of the major limiting factors for successful intervertebral disc engineering after herniation $[7,8,10,11,26]$. During the last decade, increasing knowledge and technical advancements in the field of tissue engineering have resulted in numerous promising strategies to replace or regenerate the nucleus pulposus (NP) [11, 19]. None of these advancements, however, has yet resulted in a clinically proven effective therapy [12, 24]. Since optimal regeneration of the NP should result in a restoration of the physiological high intradiscal pressure, the surrounding AF is generally of too inferior quality to withstand these forces [15]. In patients treated for disc herniation, there is often a loss of annulus tissue, restricting the potential of sutures and glues [1, 12]. These materials have limited strength, and the annulus usually has to be closed under tension. Tissue engineering strategies of the AF that deal with the 'gap' due to loss of AF tissue are currently being developed. However, the attempts are mainly directed towards 
the engineering of native AF tissue, especially in the long term, instead of providing instant mechanical strength after surgery [5]. These AF tissue engineering attempts are therefore not ready to be used in adjunct nucleus replacement strategies.

In the current study, we investigate experimental ACDs, designed to be used in adjunct to nucleus replacement therapies in a goat model. These devices are primarily intended to enable the study of nucleus replacement therapies in animal models. However, the findings may also reveal valuable information for the development of human annulus closure devices. The purpose of the implants is to provide immediate mechanical support without affecting the nucleus replacement therapy or spinal biomechanics. Biomechanical and in vivo evaluation of the ACDs were performed in a goat model, both solely and in addition to a collagen nucleus replacement matrix that has been described earlier [12, 24].

\section{Materials and methods}

\section{Nucleus replacement model}

A standardised discectomy procedure was developed, intended to allow the evaluation of novel nucleus replacement therapies in vivo. The procedure was designed for, and performed on mature Dutch female goat intervertebral discs. Initially, the NP is evacuated with custom-made instruments (Fig. 1a). These instruments consist of tubes with increasing diameter, which are used to make an entry site laterally into the AF. Via the largest tube, with an outer diameter of $3 \mathrm{~mm}$, instruments are inserted to evacuate the NP. The discectomy was always performed as complete as possible without damaging the AF or the endplates, and the result was judged by the surgeon before continuation. After evacuation, the disc space is filled with a dense collagen implant (NuRes, Arthro Kinetics AG, Esslingen, Germany) that has been described previously [6]. Shortly, collagen gel is polymerised after which the density is increased by plastic compression. The chosen density was $25 \% \mathrm{w} / \mathrm{w}$ of collagen, which has a stiffness comparable to the native NP. For this study, the collagen matrix was prepared in a 'snake'-like shape (diameter $2.5 \mathrm{~mm}$, length $30 \mathrm{~mm}$, volume $\sim 0.6 \mathrm{~cm}^{3}$ ), allowing implantation via the tubes (Fig. $1 \mathrm{~b}$ ). After insertion of the collagen implant, the annulus defect was closed with one of the four different versions of a polyethylene closure device described below.

Annulus closure devices (ACDs)

We first performed extensive preliminary testing, using the same set up for axial compression as described below (see

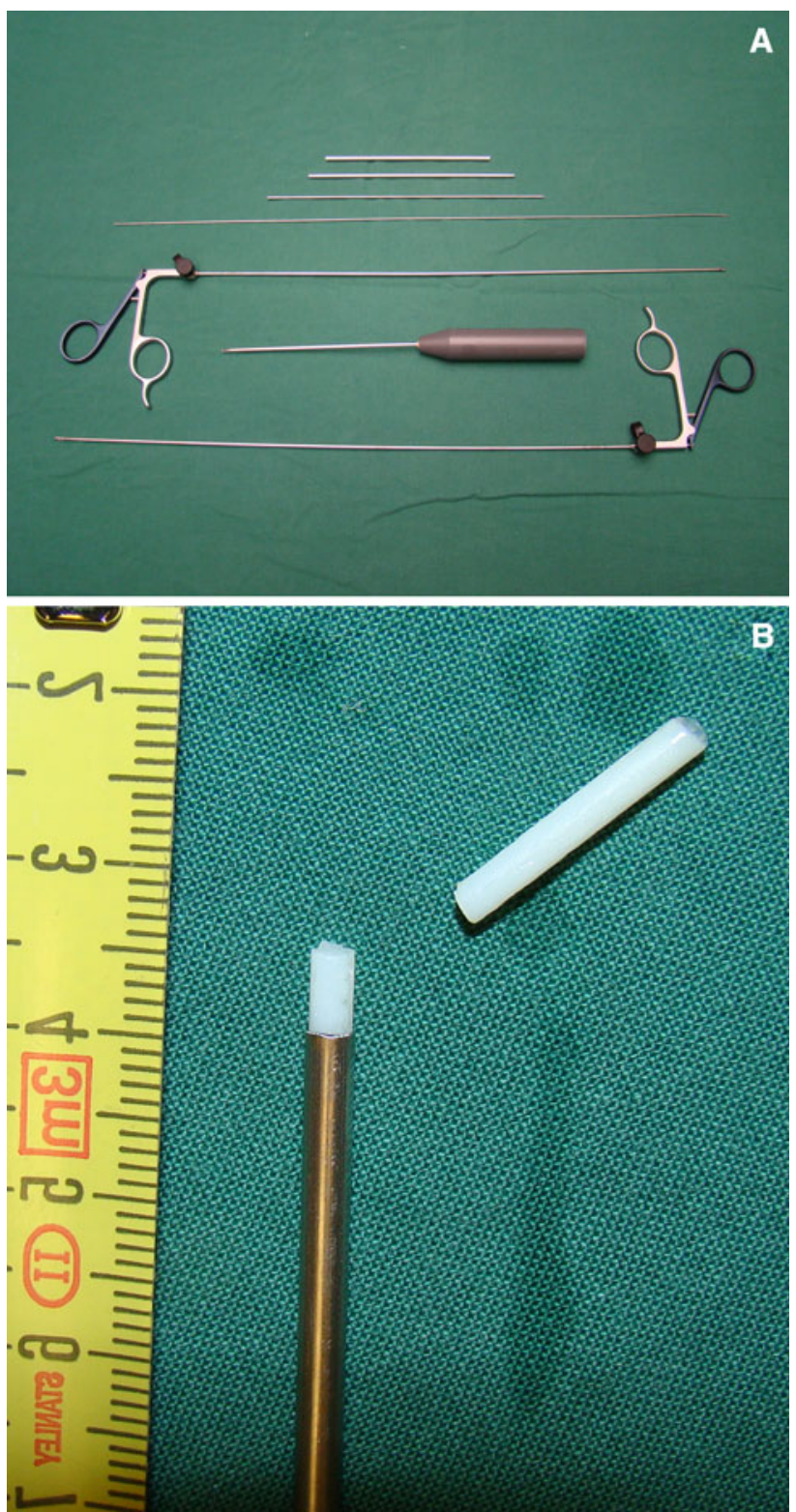

Fig. 1 Image of the instruments (a) used for evacuation of the nucleus pulposus containing rongeurs, tubes, a guide wire (for penetration of the annulus fibrosus) and a 'spoon'-like instrument. The largest tube has an outer diameter of $3 \mathrm{~mm}$ and an inner diameter of $2.5 \mathrm{~mm}$, through which the other instruments and collagen implant can be introduced. $\mathbf{b}$ The collagen implant and the end of the insertion tube

"Biomechanical evaluation"). These pilot experiments (data not shown) were intended to determine the optimal shape and dimensions of the annulus closure devices (Fig. 2a). All devices were intended to close a standardised 3-mm circular defect in the AF of the goat intervertebral disc, as described above. Four devices were further evaluated in the current study (Fig. 2b) since they were found to withstand axial compression forces over $1,000 \mathrm{~N}$. These four ACDs were composed of polyethylene and consisted 

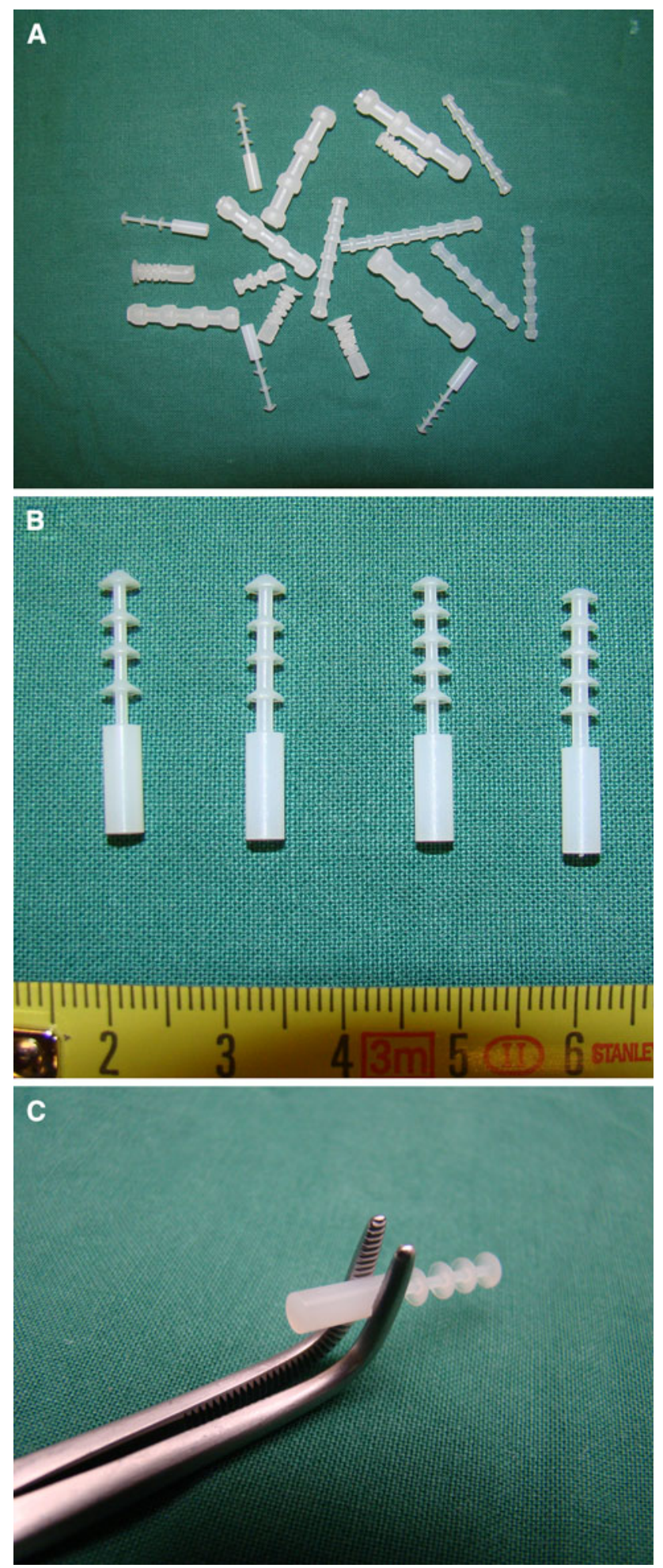

Fig. 2 Overview image of different closure systems used in the pilot experiments (a) and in current study (b). The implants vary in the number of barb rings (4 or 5) and in the diameter of the core (1.3 or $1.5 \mathrm{~mm})$. The instrument that was used to implant the devices is shown in $\mathbf{c}$ of a core (diameter 1.3 or $1.5 \mathrm{~mm}$ ) with four or five barb rings that have a maximum diameter of $3.5 \mathrm{~mm}$ (Fig. 2b). The ACDs were introduced into the AF till all barb rings were inside the defect. The back end of ACDs was used to hold the implants during implantation, and this was cut after implantation (Fig. 2c).

Intradiscal pressure calibration measurements

In order to determine the relation between the applied load and the pressure inside the goat intervertebral disc, we first performed pressure measurements. This information is essential to be sure whether the resulting pressures of the applied loads in the current study are comparable to the intradiscal pressures known from studies in vivo. For these calibration experiments, the lumbar spine (L1-L6) of a goat, derived from a local abattoir, was meticulously cleaned of soft tissues. The posterior elements were left intact. The spine was separated into three separate motion segments by incision of the discs between L2-L3 and L4L5. The ends of the vertebrae were embedded in a low melting point bismuth alloy, and the motion segments were placed in upright position in the biomechanical testing apparatus (Instron, Norwood, MA, USA). First, a pressure needle was inserted anteriorly into the core of each disc. Next, a load was applied increasing with $50 \mathrm{~N} / \mathrm{s}$ to a maximum of $1,000 \mathrm{~N}$ or a maximum of $3 \mathrm{MPa}$ as measured by the needle, whatever came first (higher pressures would result in irreversible needle damage). Both the values for load and pressure were documented. The measurements were repeated two times with the needle inserted via both lateral sides of the discs.

\section{Biomechanical evaluation}

Biomechanical experiments were performed prior to the in vivo study on spinal segments, derived from the local abattoir. Using the same set up as described with the pressure experiments, the axial failure loads of different ACDs were tested using the standardised nucleus replacement model. After the implants were inserted, an axial compression load was applied to a maximum of 5,000 N. The experiments were ended when failure, considered as the leakage of the collagen implant or extrusion of closure devices, was observed. Each ACD was tested on three different motion segments (L1-L2, L3-L4 and L5-L6). For every experiment a freshly dissected spinal segment was used.

In addition to the failure experiments, the effects of the implants on the biomechanical behaviour of the motion 
segments were investigated. These experiments were mainly performed to exclude the undesired effects on the range of latero-flexion or flexion-extension due to the ACDs and to assess the possibility of the implants to restore the effects after discectomy. After fixation in bismuth, 12 motions segments (four of each different level) were multidirectionally tested using a four-point flexion-extension set up and the instron 8872 testing machine (Instron Corp., Norwood, MA, USA). The motion segments were submitted to four cycles of flexion-extension and latero-flexion under a maximum moment of $2 \mathrm{Nm}$ at a speed of $1 \%$ s. Specimens were tested before discectomy (native), after discectomy and with both implants (nucleus implant and the four rings $1.5 \mathrm{~mm}$ ACD) inside. During all experiments, the segments were kept moisturised by wrapping with surgical gauze drowned in $0.9 \%$ saline. Force-deformation data acquisition was performed for each direction through materials testing software (Fast Track 2, Instron Corp., Norwood, MA, USA). The range of motion-data of the third cycle of the tests was used for further calculation. The mean changes in the range of motion after discectomy and implantation of both implants were calculated as ratios compared to native values (treatment over control).

\section{In vivo evaluation}

Surgical procedure and animal care were performed in compliance with the regulations of the Dutch legislation for animal research, and the Animal Ethics Committee of the VU University Medical Center approved the protocol. Ten goats were sedated with $10 \mathrm{mg} / \mathrm{kg}$ ketamine and $1.5 \mathrm{mg}$ atropine intramuscularly, followed by $0.4 \mathrm{mg} / \mathrm{kg}$ etomidate intravenously. General anaesthesia was maintained with $4 \mu \mathrm{g} / \mathrm{kg}$ fentanyl per hour, $0.3 \mathrm{mg} / \mathrm{kg}$ midazolam per hour and $1.5-2.5 \%$ isoflurane. Before surgery, standardised lateral thoracolumbar roentgenograms were obtained. A dorsal paravertebral incision was made. The IVDs were identified using a left retroperitoneal approach and exposed after mobilisation of the psoas muscle. Level determination was performed by identification of the lowest rib. Two discectomies, as described above, were randomly performed over the levels T13-L1, L2-L3 or L4-L5. At one of these levels, the collagen nucleus implant was inserted after evacuation of the NP, followed by closure of the annulus defect with the ACD (sterilised by $\gamma$-irradiation). At the other level the plug was inserted solely. Two variants of the ACDs were used in the 6-week follow-up group: four goats received implants with a core diameter of $1.3 \mathrm{~mm}$, the remainder with a core diameter of $1.5 \mathrm{~mm}$. The number (four) and diameter $(3.5 \mathrm{~mm}$ ) of the barb rings was the same for all implants.
Two of the goats were terminated by an overdose pentobarbital after 2 weeks and the remaining eight goats after 6 weeks. The latter group returned to their habitual environment from 1 week postoperative until 1 week prior to the autopsy. Evaluation was similar for all goats. After termination, the lumbar spines of the goats were harvested, and magnetic resonance imaging (MRI) of the explants was performed within $2 \mathrm{~h}$. Hereafter, all soft tissue was removed, and careful macroscopic inspection was performed. Finally, a band saw was used to obtain transversal slices of the discs for macroscopic inspection. Both, macroscopic examination and MR Imaging were used to determine the position of the ACDs. The position was classified as "in situ", partially displaced (maximum of two barb rings outside the $\mathrm{AF}$ ), or fully displaced (at least two barb rings outside the $\mathrm{AF}$ ).

\section{Statistics}

To calculate differences between different implant groups, the Student $T$ test was used.

\section{Results}

Pressure calibration measurements

The relation between the applied load and the pressure inside the three discs is shown in Fig. 3. A load of $600 \mathrm{~N}$ corresponds with an intradiscal pressure of $3 \mathrm{MPa}$. No effect was observed by changing the place of needle insertion from anterior to lateral, indicating that hydrostatic pressure was present within the entire NP.

\section{Biomechanical evaluation}

All four annulus closure devices showed a mean axial load of at least 1,000 N (Fig. 4), which equals a pressure of

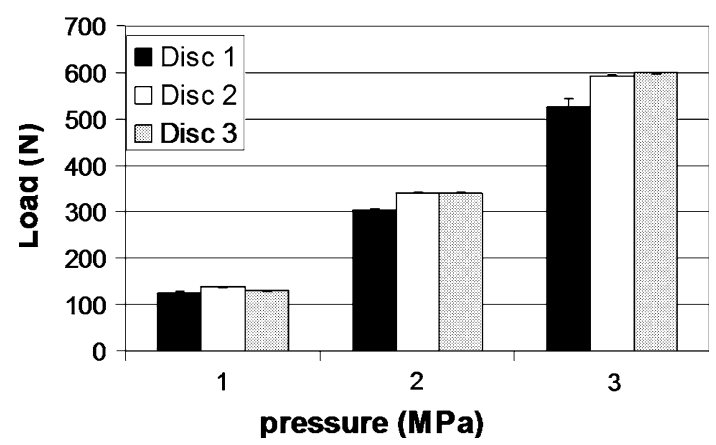

Fig. 3 Relation between the applied axial load and pressure measured inside the discs. Each bar represents the mean of three measurements: insertion of the needle via anterior or from both lateral sides 


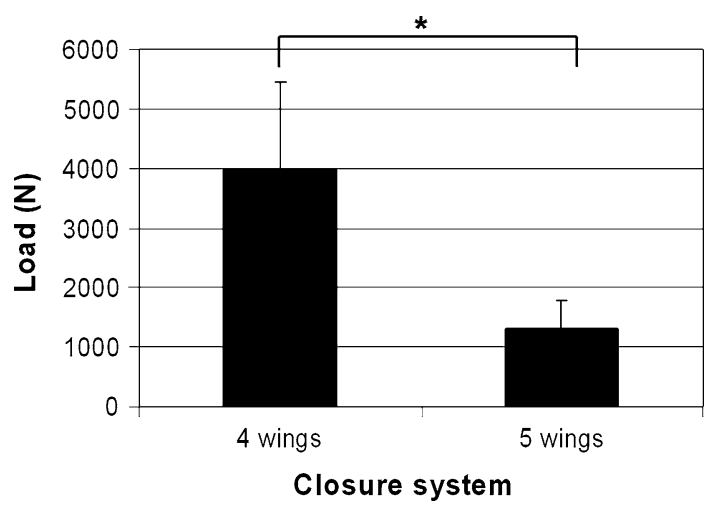

Fig. 4 Failure loads of annulus closure system with four or five barb rings. The implants with four barb rings have a significantly higher $(p<0.05)$ failure load compared to the implants with five barb rings. Each bar represents the mean of three measurements in three different segments (L1-L2, L3-L4 and L5-L6)

approximately $5 \mathrm{MPa}$ (as deducted from the results of the pressure experiments). The ACDs with four barb rings performed much better than the devices with five barb rings (Fig. 4). There was no difference in failure loads between devices with a 1.3 or 1.5 core diameter (data not shown). However, application of the devices with a $1.3-\mathrm{mm}$ core was sometimes difficult since the implants easily buckled during implantation.

The results from the flexion-extension and latero-flexion experiments are shown in Fig. 5. The bars represent the mean changes with respect to the native values (ratio). The range of both latero-flexion and flexion-extension significantly increased after the discectomy. The increase was much higher for flexion-extension (59\%) compared to latero-flexion (17\%). After implantation of the ACDs and nucleus implants, the range of motion was restored to normal values both for flexion-extension and lateroflexion.

Based on these results, revealing a sufficient high axial failure load and a lack of undesired effects of the ACD on the range of motion, continuation towards animal experiments was judged feasible, and they were thus performed.

In vivo experiments

All goats recovered well from surgery and no per- or postoperative complications were observed. Two weeks after implantation of the ACDs (all have four barb rings, $1.5-\mathrm{mm}$ core diameter), no displacement of the annulus closure devices in either animal with or without the nucleus replacement was observed. This was deduced from MR images and confirmed by macroscopic examination (Fig. 6). Based on these results, a study with longer followup was initiated. The results after 6-week follow-up were not as successful as shown in Table 1. Only two of the
Range of Motion

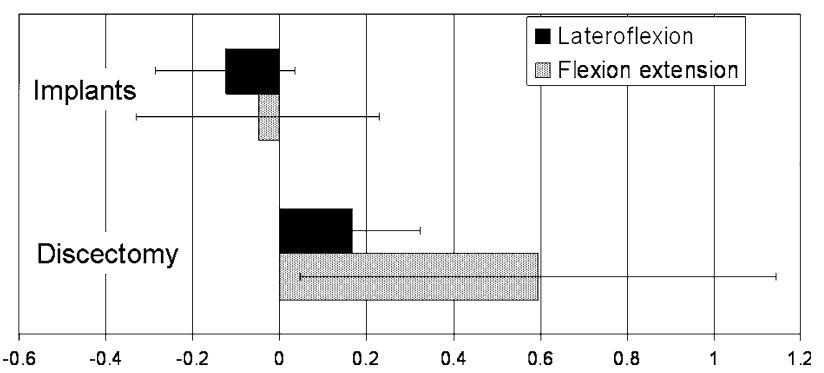

Fig. 5 Graphic showing the results of flexion-extension and lateroflexion experiments. The motion segments were tested native, after discectomy and after implantation of the implants. The values after discectomy and with the implants are shown as fractions change compared to the native values. After discectomy, a significant increase $(p<0.05)$ in both values was observed. After implantation of the nucleus and annulus implants, no significant changes compared to native values were observed. Importantly, the ACD does not significantly reduce latero-flexion

closure devices remained in situ, both in the NP replacement group (Fig. 7). Seven of the closure devices were partially displaced (less than two barb rings) and seven were fully displaced. There are no statistical differences between the groups although there is a tendency towards better results when the closure devices are combined with the collagen implants. All ACDs revealed signs of severe plastic deformation, especially of the barb rings. This is also the case for both closure devices that remained in situ (Fig. 7). Two endplate reactions were observed, irrespective whether NP replacement was performed (Fig. 8). MRI proved to be especially valuable to determine the position of fully displaced ACDs and to observe the extent of the endplate reactions. Careful examination of the MR images confirmed that the ACDs are located in the centre of the reaction in both cases (Fig. 9).

\section{Discussion}

The need for annulus closure methods in addition to nucleus replacement strategies is increasingly being recognised [3-5, 13, 20]. Wilke et al. [24] showed that a collagen scaffold allows restoration of disc height and stability after herniation in vitro. However, the absence of an appropriate annulus closure technique limits the potential and applicability in vivo. These authors also showed that glues, sutures or a combination of both are insufficient to provide sufficient containment of a collagen scaffold [12]. This agrees with the findings in scientific literature, in which an appropriate closure method has never been documented thus far $[1,4]$. In the current study, we found excellent results with experimental ACDs in vitro and after 2-week follow-up in vivo. However, after 6 weeks, the 


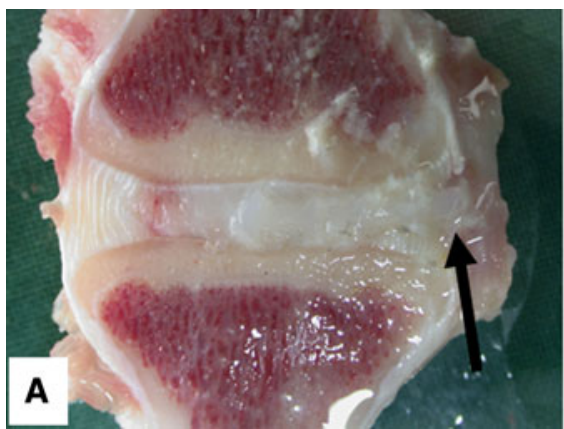

Fig. 6 Macroscopic images of two intervertebral discs treated with the annulus closure system after 2-week follow-up. a A disc treated with the addition of a collagen nucleus replacement, $\mathbf{b}$ a disc treated with the ACD solely. The arrows show the ends of the implants. The

Table 1 The results of the annulus closure devices after 6-week follow-up

\begin{tabular}{llll}
\hline & In situ & Partial displaced & Displaced \\
\hline ACD 1 & & 1 & $3^{\mathrm{a}}$ \\
ACD 2 & 1 & 3 \\
ACD $1+$ collagen & 2 & 1 & 1 \\
ACD $2+$ collagen & & $4^{\mathrm{a}}$ & \\
\hline
\end{tabular}

In situ refers to the absence of any displacement compared the implanted position. "Partial displaced" refers the displacement with a maximum of two barb rings outside the annulus fibrosus, whether "displaced" refers to a displacement of at least two wings outside the IVD

$A C D 1$ annulus closure device with $1.5 \mathrm{~mm}$ core, $A C D 2$ annulus closure device with $1.3 \mathrm{~mm}$ core

${ }^{\text {a }}$ One endplate reaction was observed in the concerning group

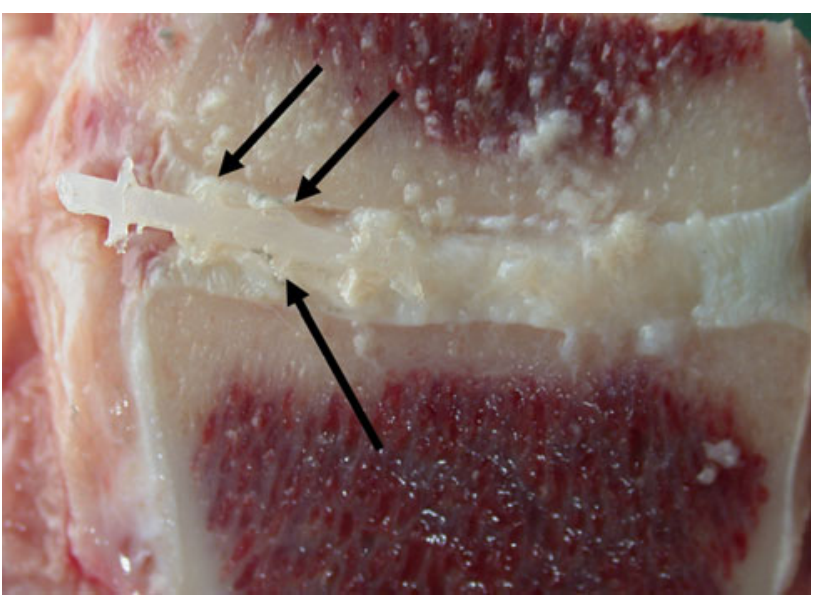

Fig. 7 Image of one of the implants that was still in situ after 6-week follow-up. There are clear signs of destruction visible at the barb rings of the implant (arrows)

majority of the implants showed signs of migration and deformation. Since the barb rings should gain adhesion into the layers of the annulus, it is not surprising that their remnants of NP tissue are very much swollen by the water used to cool the sawing blade. Due to the sawing, the collagen implant has also been washed out, and is therefore not visible in $\mathbf{a}$
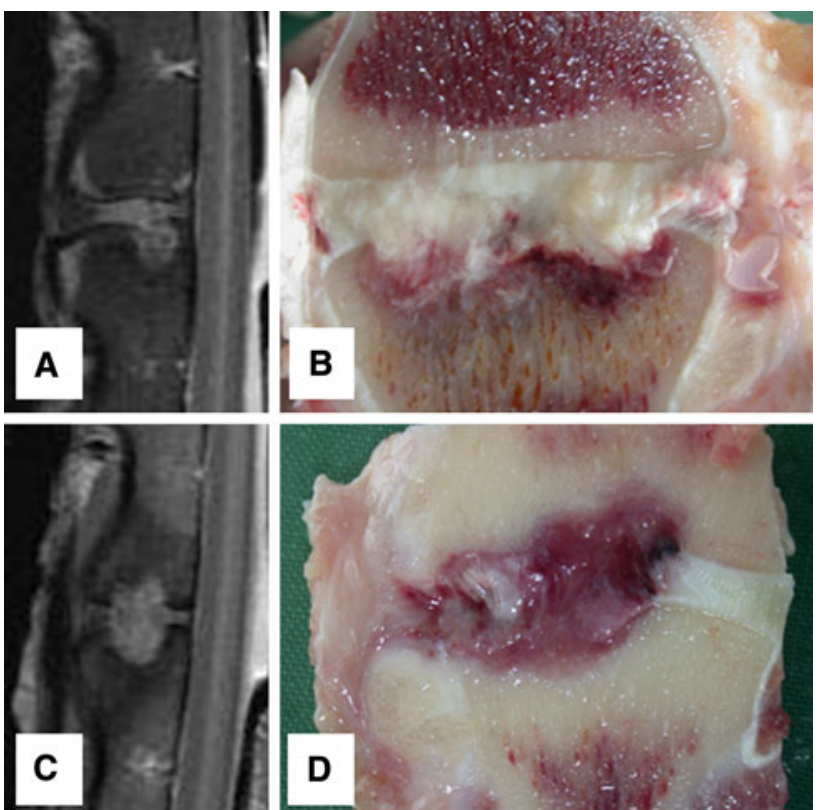

Fig. 8 MRI and macroscopic images of the two levels in which endplate reaction were encountered. Images $\mathbf{a}$ and $\mathbf{b}$ are of a level that was treated with an annulus closure system solely, and the reaction is mainly located at a single endplate. Images $\mathbf{c}$ and $\mathbf{d}$ are of a level treated with the closure system combined with the collage nucleus replacement implant. The reaction in this goat is located at both endplates and extends into the subchondral space

destruction results in implant extrusion. The ACDs in the goats after 2-week follow-up showed only mild signs of damage. During these 2 weeks the goats stayed in the shed of the university animal facilities and are recovering from the surgeries. In this period, the animals might behave more quiescent than they usually do. Between the second and sixth week after the surgeries, the goats are fully recovered. The animals return to the farm and regain their usual activities. This may result in increasing forces on the implants, and when the damage to the barb rings accumulates to a certain level, the implants start to displace. 


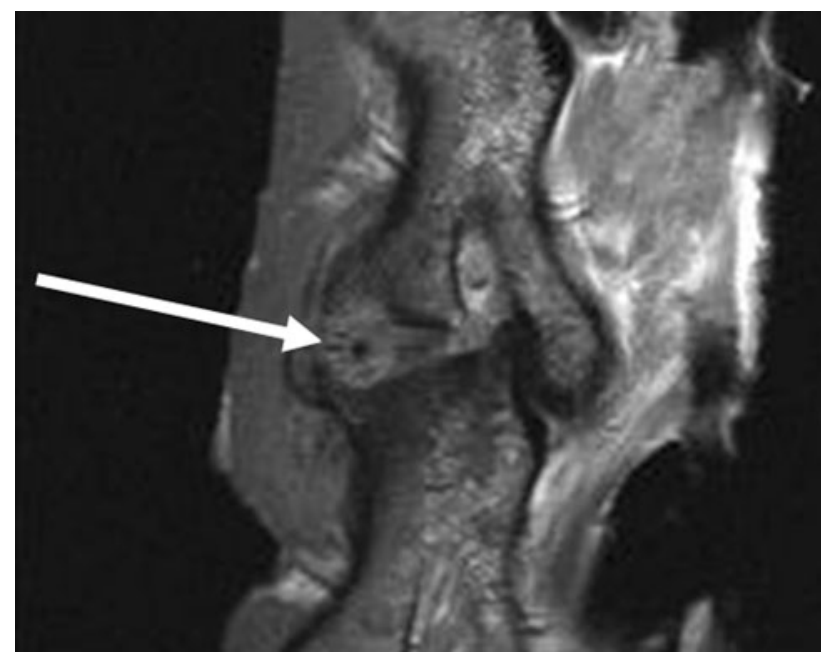

Fig. 9 MRI image that shows that the annulus closure system is located in the centre of the endplate reaction (arrow), suggesting a causative role

The former agrees with the finding that those implants that were fully extruded were also the most damaged ones. The damage should have occurred prior to the moment of extrusion since the implants are located in the soft tissue directly adjacent to the disc, where no direct mechanical stresses are encountered.

Two of the implants even provoked a severe osteolytic reaction extending through the endplates into the subchondral bone. This reaction was observed in both groups, with and without a collagen implant, and it seems therefore not likely that the reaction was caused by the latter. Most probably the reactions are the result of the friction described above, which has resulted in pressure necrosis of the endplates. Another option, however less likely, may be that the endplates were damaged during surgery, and that leakage of nucleus material into the subchondral bone provoked the reaction. The potential for nucleus material, which normally does not encounter immune reactive cells due to the absent vascularisation in the disc space, to initiate such a reaction has been hypothesised earlier [2].

To prevent failure and endplate reactions, the design, dimensions and stiffness of the closing devices can be altered. Current devices were fabricated from polyethylene, which was used for its known biocompatibility. Polyethylene, however, is a rather stiff material, and friction between the barb rings and endplates might therefore have resulted in the observed reaction [18]. Taking a less stiff material would have decreased the risk, but might probably result in lower mechanical expulsion strength. The shape of the implants, especially of the barb rings, deserves attention in further optimisation of the closure devices. We performed several pilot experiments with different designed devices, but the current shape showed the highest resistance to forces. We do not know, however, why implants with four barb rings performed so much better than implants with five barb rings. The dimensions of the closure devices can also be adjusted. Intradiscal pressure results in forces on the implants that are dependent of diameter and length. We always made a standardised circular defect of $3 \mathrm{~mm}$ in the annulus, and the diameter of the barb rings of the implants was $3.5 \mathrm{~mm}$. The ring diameter was chosen to promote adherence between the annulus layers. A larger diameter would have increased this adherence, but would also have resulted in larger expulsion forces from the pressurised NP and subsequent failure at lower axial compression forces. The expulsion forces on the ACD depend on its cross-sectional diameter, whereas the friction forces only depend on the circumference. In addition, regarding the disc height of the goat $(4-5 \mathrm{~mm})$, a larger diameter could also result in continuous contact between the implant and both endplates, increasing the risk of adverse reactions and ring damage.

Although current biomechanical study results did not show a significant effect on latero-flexion, a tendency towards some small restriction could already be observed. Decreasing the diameter of both the defect and barb rings might therefore have been preferred from a mechanical viewpoint and from the aim to reduce contact with the endplate. Unfortunately, this was currently not possible regarding the diameter of the nucleus implants. The length of the ACDs was maximal $15 \mathrm{~mm}$ (between front of the first and the back of the last barb ring), and this length was chosen since it always covers the whole lateral annulus. For some goats, however, a smaller length would have been sufficient, but this can only be judged afterwards at macroscopic evaluation. If current closure systems would have passed the animal experiments, a decrease of the lengths to allow more space for the nucleus replacements could have been evaluated. The main goal of the biomechanical experiments was to predict failures of the ACDs. We did not study torsion since the forces would be mainly distributed through the facet joints with only a marginal increase of the intradiscal pressure [21].

We found an axial load of $600 \mathrm{~N}$ to correspond to an intradiscal pressure of approximately $3 \mathrm{MPa}$. The applied load will be partially distributed through the annulus parts of the discs and/or posterior elements. We performed these experiments to allow comparison of the forces known from pressure measurements in vivo. A few studies have been performed measuring the pressure inside the human intervertebral disc in vivo [16, 17]. Wilke et al. [25] found a maximum intradiscal pressure of $2.3 \mathrm{MPa}$ during the lifting of $20 \mathrm{~kg}$ combined with flexion-extension forward. From our own in vivo measurements in goats we know that the axial load can rise up to $900 \mathrm{~N}$ [9]. According to current pressure measurements, this would correspond to a 
pressure over $4 \mathrm{MPa}$. Thus, the peak pressure inside the goat intervertebral disc seems to be higher than the peak pressure inside the human disc. This finding agrees with the fact that the bone density of the vertebra of goats is higher compared to humans, indicating higher stresses in vivo [23]. The differences might be explained by the fact that the forces on the goat spine are generated by muscles and ligaments surrounding the continuously bended spine. In the bipedal situation of humans, these compressive forces are lower and more dependent on activity and posture [14]. Furthermore, we used motion segments derived from young goats (age 4 years), and the osmotic pressure might therefore be higher compared to the adult human disc [22]. The goat model was used, since prior studies have shown that the absolute spinal forces in this animal are still comparable to that of humans $[12,23]$. The mean value of axial load of 4,000 N, which we found that the current annulus closure system could withstand, provides a sufficient safety range.

A limitation of the current study is that we only performed maximum axial failure load testing prior to implantation and no duration testing. Given the short-term in vivo results, however, this would not have forecasted the failures. The number and multi-directionality of biomechanical test loadings that should be applied to match to the goat spines during 6 weeks in vivo will not easily be obtained in vitro. Current ACDs are intended to allow evaluation of novel nucleus replacement therapies in animal models, and the results cannot easily be extrapolated to closure techniques of the $\mathrm{AF}$ in patients with disc herniation undergoing a discectomy. Current discectomy procedure was performed in a very standardised manner in healthy discs with fixed location and size in the lateral AF. This is in contrast to the human situation after a discectomy, where a very variable amount of the AF is damaged at the thin posterolateral part of the AF. For human AF closure, which should ultimately accompany a potential successful NP replacement, other closure techniques should be developed [5].

In conclusion, the current study found encouraging results with a novel annulus closure system in vitro and after 2 weeks in vivo. After 6-week follow-up, however, most implants revealed signs of severe plastic deformation and subsequent displacement. Although there was a tendency towards better results when combined with a nucleus replacement, these differences were not statistically significant. Further research on annulus closure devices, in order to allow the in vivo evaluation of nucleus replacement therapies, is therefore indicated. Current results also illustrate the importance of in vivo confirmation of results obtained by biomechanical experiments, especially in the field of annulus closure techniques.
Acknowledgments This study was supported by Arthro Kinetics AG (Esslingen am Neckar, Germany). The authors like to thank Klaas Walter Meyer, Paul Sinnige (both from the Department of Animal Experiment), Wouter Jurgens, MD (Department of Plastic and Reconstructive Surgery) and Robert Jan Kroeze, MD (Department of Oral Cell Biology) for their assistance in the surgeries and/or autopsies. Ger Vink and Jan Blom are acknowledged for taking care of the goats.

Open Access This article is distributed under the terms of the Creative Commons Attribution Noncommercial License which permits any noncommercial use, distribution, and reproduction in any medium, provided the original author(s) and source are credited.

\section{References}

1. Ahlgren BD, Lui W, Herkowitz HN, Panjabi MM, Guiboux JP (2000) Effect of anular repair on the healing strength of the intervertebral disc: a sheep model. Spine 25(17):2165-2170

2. Albert HB, Kjaer P, Jensen TS et al (2008) Modic changes, possible causes and relation to low back pain. Med Hypotheses 70(2):361-368

3. Bajanes G, Perez A, Diaz M (2007) One year follow up of discectomy patients who received a mesh to repair the annulus fibrosus, vol 7. Spine Arthroplasty Society, Berlin

4. Bourgeault C, Beaubien B, Griffith S (2007) Biomechanical assessment of annulus fibrosus repair with suture tethered anchors, vol 7. Spine Arthroplasty Society, Berlin

5. Bron JL, Helder MN, Meisel HJ, van Royen BJ, Smit TH (2009) Repair, regenerative and supportive therapies of the annulus fibrosus: achievements and challenges. Eur Spine J 18(3):301-313

6. Bron JL, Koenderink GH, Everts V, Smit TH (2009) Rheological characterization of the nucleus pulposus and dense collagen scaffolds intended for functional replacement. J Orthop Res 27(5):620-626

7. Carragee EJ, Han MY, Suen PW, Kim D (2003) Clinical outcomes after lumbar discectomy for sciatica: the effects of fragment type and anular competence. J Bone Joint Surg Am 85-A(1):102-108

8. Choy DS (2000) Familial incidence of intervertebral disc herniation: an hypothesis suggesting that laminectomy and discectomy may be counterproductive. J Clin Laser Med Surg 18(1):29-32

9. Dormans KW, Krijnen MR, Geertsen S, van Essen GJ, Wuisman PI, Smit TH (2004) Telemetric strain measurements in an interbody fusion cage: a pilot goat study. In: Proceedings of the 14th European Society of Biomechanics (ESB) conference. 's-Hertogenbosch, Netherlands, p 224

10. Hansson E, Hansson T (2007) The cost-utility of lumbar disc herniation surgery. Eur Spine J 16(3):329-337

11. Hegewald AA, Ringe J, Sittinger M, Thome C (2008) Regenerative treatment strategies in spinal surgery. Front Biosci 13:15071525

12. Heuer F, Ulrich S, Claes L, Wilke HJ (2008) Biomechanical evaluation of conventional anulus fibrosus closure methods required for nucleus replacement. Laboratory investigation. J Neurosurg Spine 9(3):307-313

13. Kamaric E, Gorensek M, Vilendecic M, Eustacchio S, Trummer M, Eskinja N, Ledic D, Yeh O, Einhorn J, Lambrecht G (2006) Surgical factors affecting reherniation rate after lumbar microdiscectomy: effect of defect size and amount of disc removed. Spine J 6(5 Suppl 1):38S 
14. Ledet EH, Tymeson MP, DiRisio DJ, Cohen B, Uhl RL (2005) Direct real-time measurement of in vivo forces in the lumbar spine. Spine J 5(1):85-94

15. Melrose J, Smith SM, Little CB et al (2008) Recent advances in annular pathobiology provide insights into rim-lesion mediated intervertebral disc degeneration and potential new approaches to annular repair strategies. Eur Spine J 17(9):1131-1148

16. Nachemson A (1965) The effect of forwards leaning on lumbar intradiscal pressure. Acta Orthop Scand 35:314-328

17. Nachemson A, Morris JM (1964) In vivo measurements of intradiscal pressure. Discometry, a method for the determination of pressure in the lower lumbar discs. J Bone Joint Surg Am 46:1077-1092

18. Ries MD, Pruitt L (2005) Effect of cross-linking on the microstructure and mechanical properties of ultra-high molecular weight polyethylene. Clin Orthop Relat Res 440:149-156

19. Sebastine IM, Williams DJ (2007) Current developments in tissue engineering of nucleus pulposus for the treatment of intervertebral disc degeneration. Conf Proc IEEE Eng Med Biol Soc 1:6400-6405

20. Sherman J, Cauthen J, Griffith S (2007) Pre-clinical evaluation of a mesh device for repairing the annulus fibrosus. Spine Arthroplasty society, Berlin
21. Shirazi-Adl A, Ahmed AM, Shrivastava SC (1986) Mechanical response of a lumbar motion segment in axial torque alone and combined with compression. Spine 11(9):914-927

22. Sivan S, Merkher Y, Wachtel E, Ehrlich S, Maroudas A (2006) Correlation of swelling pressure and intrafibrillar water in young and aged human intervertebral discs. J Orthop Res 24(6):12921298

23. Smit TH (2002) The use of a quadruped as an in vivo model for the study of the spine-biomechanical considerations. Eur Spine J 11(2):137-144

24. Wilke HJ, Heuer F, Neidlinger-Wilke C, Claes L (2006) Is a collagen scaffold for a tissue engineered nucleus replacement capable of restoring disc height and stability in an animal model? Eur Spine J 15(Suppl 3):S433-S438

25. Wilke HJ, Neef P, Caimi M, Hoogland T, Claes LE (1999) New in vivo measurements of pressures in the intervertebral disc in daily life. Spine 24(8):755-762

26. Yeung AT, Yeung CA (2007) Minimally invasive techniques for the management of lumbar disc herniation. Orthop Clin North Am 38(3):363-372 